\title{
Contrary Behavior of Absorption and Dispersion
}

\author{
Katrin Dahl, Luca Spani Molella, Rolf-Hermann Rinkleff, and Karsten Danzmann \\ Albert Einstein Institute, Max Planck Institute for Gravitational Physics, and Institut für Gravitationsphysik, \\ Gottfried Wilhelm Leibniz Universität Hannover, Callinstraße 38, D-30167 Hannover, Germany. \\ Authore-mail address: katrin.dahl@aei.mpg.de
}

\begin{abstract}
A degenerate two-level system was investigated with circularly polarized probe and coupling lasers. An intensity dependent switch of an absorption peak to a dip was measured. The corresponding dispersion did not change.

(C) 2009 Optical Society of America

OCIS codes: (020.1670) Coherent optical effects; (130.4815) Optical switching devices
\end{abstract}

\section{Introduction}

Electromagnetic induced absorption (EIA) in a degenerate two-level system has been investigated with a probe laser and coupling laser of different polarization combinations. For counter-rotating lasers of circular polarization an intensity dependent switch from "absorption within transparency" to "transparency within transparency" is observed [1]. In conditions of "absorption within transparency" a broad absorption dip in the coupling laser spectra is generated through the interaction between probe laser and coupling laser. At the two-photon resonance a much narrower absorption peak is superimposed to the broad dip (see Fig. 1.). If this extra peak changes its sign and becomes an extra transparency dip one speaks of "transparency within transparency".

We completed these findings concerning the intensity dependent absorption behavior of $\sigma-\sigma$ polarized lasers and measured the corresponding dispersion spectra which to our knowledge has not been detected so far.

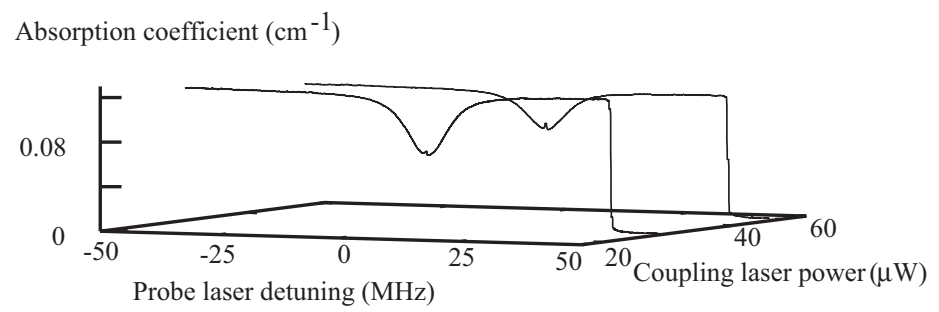

Fig. 1. Coupling laser absorption spectra: probe laser power $(0.038 \mathrm{~mW})$ is below the saturation power.

\section{Experiment}

With a probe laser beam and coupling laser beam of counter-rotating circular polarizations, the absorption and dispersion in a medium typically showing EIA was studied. All measurements were performed within the $\mathrm{D}_{2}$ line of cesium, on the hyperfine transition $6 \mathrm{~s}^{2} \mathrm{~S}_{1 / 2}, \mathrm{~F}=4$ to $6 \mathrm{p}^{2} \mathrm{P}_{3 / 2}, \mathrm{~F}=5$. An atomic beam perpendicular to the direction of propagation of the lasers was used to minimize the Doppler broadening of the signals. A more detailed description of the experimental setup can be found in $[2,3]$.

For counter-rotatingly polarized laser beams we measured coupling laser absorption spectra as a function of the coupling laser power, below and above the saturation power of the atomic sample. In the case of a probe laser power below saturation for all coupling laser powers, "absorption within transparency" was registered exclusively (see Fig. 1.). By contrast, above saturation a switch from "absorption within transparency" to "transparency within transparency" was detected when the coupling laser power was larger than the constant probe laser power (see Fig. 2.). Interestingly, the corresponding dispersion spectra of the coupling laser remained positive (see Fig. 3.). In other words, opposed to the absorption spectra no switch was observed. This characteristic of the dispersion agrees well with the theoretical predictions given in [4]. 


\section{JWA101.pdf}

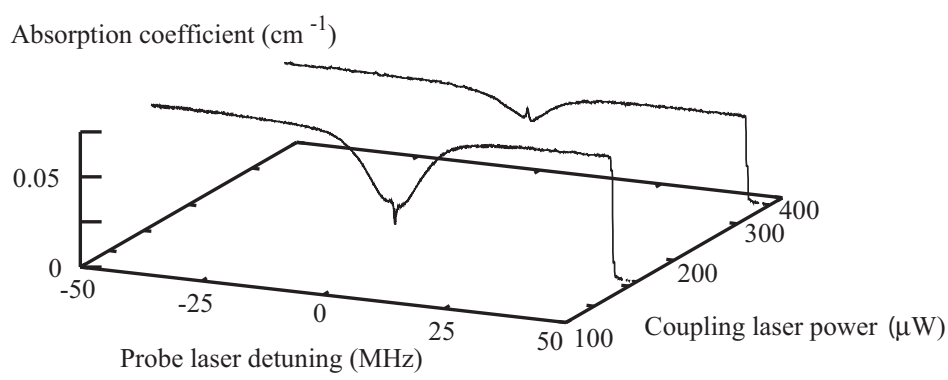

Fig. 2. Coupling laser absorption spectra: probe laser power $(0.252 \mathrm{~mW})$ is above the saturation power.
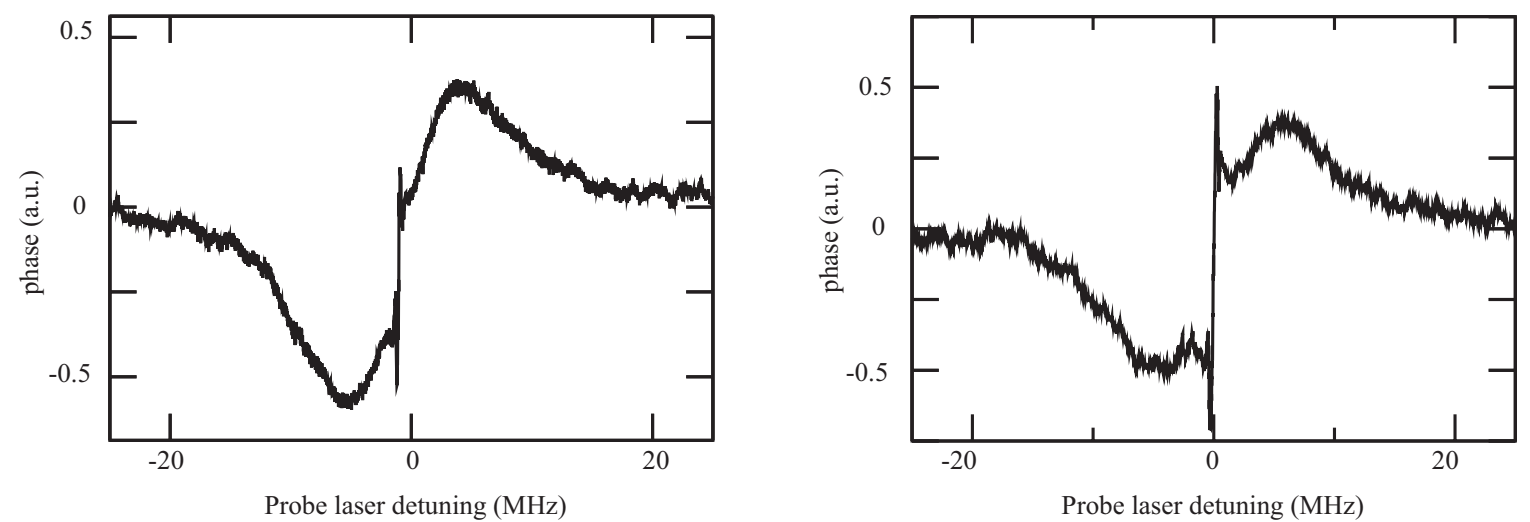

Fig. 3. Coupling laser dispersion in arbitrary units: left: coupling laser power $(0.044 \mathrm{~mW})$ is below the saturation power; probe laser power $0.102 \mathrm{~mW}$; right: coupling laser power $(0.134 \mathrm{~mW})$ is above the saturation power, probe laser power $0.160 \mathrm{~mW}$.

\section{Acknowledgements}

The work was made possible through the financial support of the Collaborative Research Centre SFB407 of the German Research Foundation (Deutsche Forschungsgemeinschaft).

\section{References}

[1] K.Dahl, L. Spani Molella, R.-H. Rinkleff, and K. Danzmann, "Switching from "absorption within transparency" to "transparency within transparency" in an electromagnetically induced absorption dominated transition," Opt. Lett. 33, 983-985 (2008).

[2] L. Spani Molella, R.-H. Rinkleff, and K. Danzmann, "Role of the coupling laser in electromagnetically induced transparency", Phys Rev. A 72, 041802(R) 1-4 (2005).

[3] L. Spani Molella, R.-H. Rinkleff, and K. Danzmann, "Spectral measurement of the caesium $\mathrm{D}_{2}$ line with a tunable heterodyne interferometer," Spectrochim. Acta A 63, 987-993 (2006).

[4] C. Goren, A.D. Wilson-Gordon, M. Rosenbluh, and H. Friedmann, “Atomic four-level N system," Phys. Rev. A 69, 053818 1-10 (2004). 